\title{
PHƯƠNG PHÁP TÌM KIẾM CÁC TRI ĐO THÔ TRONG QUÁ TRİNH BÌNH SAI MẠNG LƯỚI TRẮC ĐỊA MẠTT BẰNG CÓ DẠNG ĐƯờNG CHUYỀN
}

\author{
ThS. LƯO'NG THANH THACH \\ Trường Đại học Tài nguyên và Môi trường Hà Nội
}

\section{Tóm tắt:}

Hiện nay, với sự phát triển của công nghệ đo đạc bản đồ, tiêu biểu là sự có mặt của công nghệ GNSS (Global Navigation Satellite System) đã giải quyết được rất nhiều những khó khăn của công nghệ đo đạc truyền thống, ví dụ như đo đạc thành lập lưới khống chế trắc địa, đo vẽ chi tiết thành lập bản đồ... Tuy nhiên, thực tế khi triển khai công việc đo đạc thành lập lưới khống chế trắc địa không phải khi nào cũng có thể ứng dụng công nghệ GNSS và áp dụng các đồ hình lưới có kết cấu chặt chẽ, thay vào đó là dùng máy toàn đạc điện tử hoặc máy kinh vĩ để thành lập các dạng lưới khống chế trắc địa dạng đơn giản như Iưới đường chuyền, mà cụ thể là lưới đường chuyền phù hợp và đường chuyền khép kín do tính "linh hoạt" của nó. Bài báo này trình bày cơ sở lý thuyết phương pháp tìm kiếm và phát hiện các trị đo thô trong lưới đường chuyền và tiến hành tính toán thực nghiệm đối với hai dạng đồ hình cơ bản trên.

\section{1. Đặt vấn đề}

Mạng lưới đường chuyền có lượng trị đo dư ít nên không thể tìm kiếm các trị đo thô một cách tin cậy theo phương pháp bình phương nhỏ nhất lặp. Về nguyên tắc, không thể tìm kiếm trị đo thô khi chỉ có một trị đo dư. Khi mạng lưới có hai trị đo dư trở lên thì việc tìm kiếm trị đo thô chỉ thực hiện được trong trường hợp trị đo thô là trị đo chung trong hai phương trình điều kiện của mạng lưới. Với nguyên tắc nêu trên, chúng ta sẽ nghiên cứu phương pháp tìm kiếm các trị đo thổ trong mạng lưới đường chuyền.

Trong quá trình bình sai truy hồi mạng lưới đường chuyền, khi phát hiện sự có mặt của trị đo thô, chúng ta tiến hành tìm kiếm trị đo thô. Quá trình tìm kiếm trị đo thô được thực hiện theo các đồ hình đường chuyền cơ bản (xem các hình 1,2 ở dưới đây). Ở hai đồ hình cơ bản này, mỗi đồ hình chỉ có 3 trị đo dư, do vậy chỉ có thể có tối đa 3 phương trình điều kiện, bao gồm hai phương trình điều kiện tọa độ và một phương trình điều kiện phương vị trong mỗi đồ hình trên để tìm kiếm được một trị đo thô (nếu có), trị đo thô trong các trường hợp này có thể là một góc đo thô hoặc một cạnh đo thô. Tuy nhiên, đối với phương pháp nghiên cứu này lại không có hiệu quả đối với mỗi đồ hình đường chuyền có nhiều hơn 1 trị đo thô, do đó sẽ không được áp dụng. Phương pháp bình sai truy hồi có ưu điểm là phát hiện sự có mặt của các trị đo thô. Đối với việc tìm hiểu các trị đo thô chúng ta sẽ nghiên cứu phương pháp được đề xuất trong [2].

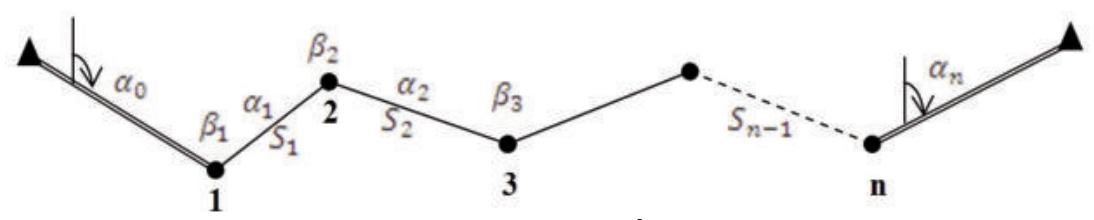

Hình 1: Đường chuyền phù hợp

Người phản biện: TS. Nguyễn Đình Thành 


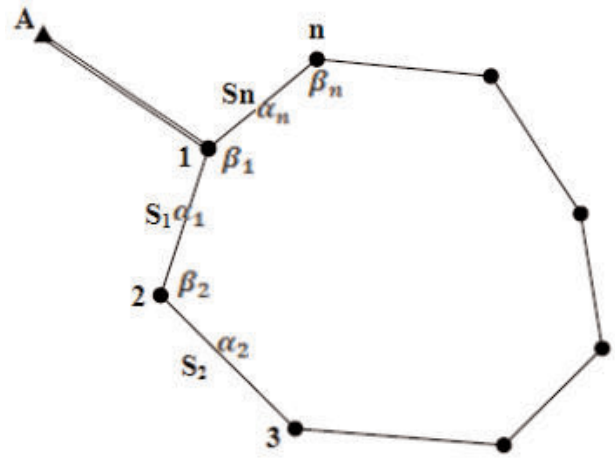

Hình 2: Đường chuyền khép kín

Giữa tuyến đường chuyền phù hợp và đường chuyền khép kín có sự khác nhau ở các mặt sau:

Tuyến đường chuyền phù hợp có $n$ góc đo và $n-1$ cạnh đo, điểm đầu (điểm 1 ) và điểm cuối (điểm $\mathrm{n}$ ) của đường chuyền đều là điểm gốc. $\alpha_{0}$ và $\alpha_{n}$ là các phương vị gốc của cạnh gốc, $\alpha_{1}, \alpha_{2}, \ldots \alpha_{n-1}$ là các góc phương vị của các cạnh đường chuyền; $\mathrm{S}_{1}, \mathrm{~S}_{2}, \ldots ., \mathrm{S}_{\mathrm{n}-1}$ - là các cạnh đo của cạnh đường chuyền; $\beta_{1}, \beta_{2}, \ldots, \beta_{n}$ - là các góc đo của tuyến đường chuyền.

Tuyến đường chuyền khép kín có $\mathrm{n}$ góc đo và $\mathrm{n}$ cạnh đo. Điểm đầu và điểm cuối là 1 . Để chuyền phương vị vào các cạnh của đồ hình sử dụng điểm đo nối phương vị ( ký hiệu là điểm $\mathrm{A}$ ). Các tọa độ phẳng $\mathrm{x}, \mathrm{y}$ của điểm 1 và điểm $\mathrm{A}$ là các tọa độ khái lược của các điểm này; $\quad \alpha_{1}, \alpha_{2}, \ldots, \alpha_{n} \quad$ - các góc phương vị của các cạnh đường chuyền; $\mathrm{S}_{1}, \mathrm{~S}_{2}, \ldots$, $\mathrm{S}_{\mathrm{n}}$ - các cạnh đo của các cạnh đường chuyền; $\beta_{1}, \beta_{2}, \ldots, \beta_{n}$ - các góc đo trong tuyến đường chuyền.

\section{Giải quyết vấn đề}

Quá trình tìm kiếm các trị đo thô trong các đồ hình đường chuyền cơ bản được thực hiện như sau:

Bước 1. Tính sai số khép phương vị

1. Đối với đường chuyền phù hợp $W_{\alpha}=\sum_{i=1}^{n} \beta_{i} \pm(n-1) \cdot 180^{\circ}+\alpha_{0}-\alpha_{n}$

2. Đối với đa giác đường chuyền khép kín $W_{\alpha}=\sum_{i=1}^{n} \beta_{i}-(n-2) \cdot 180^{\circ}$

Bước 2. Tính các số gia tọa độ của các cạnh và tọa độ của các điểm theo chiều thuận và chiều nghịch

1. Đối với đường chuyền phù hợp

* Tính các số gia tọa độ theo chiều thuận:

$$
\begin{aligned}
& S_{1} \cdot \cos \alpha_{1} ; S_{2} \cdot \cos \alpha_{2} ; \ldots ; S_{n-1} \cdot \cos \alpha_{n-1} \\
& S_{1} \cdot \sin \alpha_{1} ; S_{2} \cdot \sin \alpha_{2} ; \ldots ; S_{n-1} \cdot \sin \alpha_{n-1}
\end{aligned}
$$

* Tính các tọa độ của các điểm đường chuyền theo chiều thuận: 


$$
\begin{aligned}
& \left(x_{i+1}\right)_{T}=\left(x_{i}\right)_{T}+S_{i} \cdot \cos \alpha_{i} \\
& \left(y_{i+1}\right)_{T}=\left(y_{i}\right)_{T}+S_{i} \cdot \sin \alpha_{i}
\end{aligned}
$$

với $\mathrm{i}=1,2, \ldots, \mathrm{n}-1$.

* Tính các số gia tọa độ theo chiều nghịch:

$$
\begin{gathered}
S_{n-1} \cdot \cos \left(\alpha_{n-1} \pm 180^{\circ}\right) ; S_{n-2} \cdot \cos \left(\alpha_{n-2} \pm 180^{\circ}\right) ; S_{1} \cdot \cos \left(\alpha_{1} \pm 180^{\circ}\right) \\
S_{n-1} \cdot \sin \left(\alpha_{n-1} \pm 180^{\circ}\right) ; S_{n-2} \cdot \sin \left(\alpha_{n-2} \pm 180^{\circ}\right) ; S_{1} \cdot \sin \left(\alpha_{1} \pm 180^{\circ}\right)
\end{gathered}
$$

* Tính các tọa độ của các điểm đường chuyền theo chiều nghịch:

$$
\begin{aligned}
& \left(x_{i-1}\right)_{N}=\left(x_{i}\right)_{N}+S_{i-1} \cdot \cos \left(\alpha_{i-1} \pm 180^{\circ}\right) \\
& \left(y_{i-1}\right)_{N}=\left(y_{i}\right)_{N}+S_{i-1} \cdot \sin \left(\alpha_{i-1} \pm 180^{\circ}\right)
\end{aligned}
$$

với $\mathrm{i}=\mathrm{n}, \mathrm{n}-1, \ldots, 2$;

2. Đối với đường chuyền khép kín

* Tính các số gia tọa độ theo chiều thuận:

$$
\begin{aligned}
& S_{1} \cdot \cos \alpha_{1} ; S_{2} \cos \alpha_{2} ; \ldots ; S_{n} \cdot \cos \alpha_{n} ; \\
& S_{1} \cdot \sin \alpha_{1} ; S_{2} \sin \alpha_{2} ; \ldots ; S_{n} \cdot \sin \alpha_{n} ;
\end{aligned}
$$

* Tính các tọa độ của các điểm đường chuyền theo chiều thuận:

với $\mathrm{i}=1,2, \ldots, \mathrm{n}-1$

$$
\begin{aligned}
& \left(x_{i+1}\right)_{T}=\left(x_{i}\right)_{T}+S_{i} \cdot \cos \alpha_{i} \\
& \left(y_{i+1}\right)_{T}=\left(y_{i}\right)_{T}+S_{i} \cdot \sin \alpha_{i}
\end{aligned}
$$

Lưu ý: Điểm $\mathrm{n}+1$ tương ứng với điểm 1

* Tính các gia số tọa độ theo chiều nghịch

$$
\begin{gathered}
S_{n} \cdot \cos \left(\alpha_{n} \pm 180^{\circ}\right) ; S_{n-1} \cdot \cos \left(\alpha_{n-1} \pm 180^{\circ}\right) ; S_{1} \cdot \cos \left(\alpha_{1} \pm 180^{\circ}\right) \\
S_{n} \cdot \sin \left(\alpha_{n} \pm 180^{\circ}\right) ; S_{n-1} \cdot \sin \left(\alpha_{n-1} \pm 180^{\circ}\right) ; S_{1} \cdot \sin \left(\alpha_{1} \pm 180^{\circ}\right)
\end{gathered}
$$

* Tính các tọa độ của điểm đường chuyền theo chiều nghịch:

$$
\begin{aligned}
& \left(x_{i-1}\right)_{N}=\left(x_{i}\right)_{N}+S_{i-1} \cdot \cos \left(\alpha_{i-1} \pm 180^{\circ}\right) \\
& \left(y_{i-1}\right)_{N}=\left(y_{i}\right)_{N}+S_{i-1} \cdot \sin \left(\alpha_{i-1} \pm 180^{\circ}\right)
\end{aligned}
$$

với $\mathrm{i}=\mathrm{n}+1, \mathrm{n}, \ldots, 2$ 
Lưu ý: Điểm n+1 tương ứng với điểm 1

Bước 3. Kiểm tra sự có mặt của trị đo thô

Hạn sai của sai số khép phương vị $\left(W_{\alpha}\right)_{c p}= \pm t \cdot m_{\beta} \cdot \sqrt{n}$, ở đây hệ số nhân t nhận bằng 2.5; còn $m_{\beta}$ - sai số trung phương đo góc được xác định một cách tiên nghiệm [2].

Nếu $W_{\alpha}<\left(W_{\alpha}\right)_{c p}$, thì các góc đo không có góc đo thô và chúng ta chuyển sang bước 4 . Trong trường hợp sai số khép phương vị không nhỏ hơn hạn sai, chúng ta tiến hành tìm kiếm góc đo thô như sau:

Tại điểm đường chuyền i có đo góc tiến hành tính các hiệu theo các tọa độ của các điểm được tính theo chiều thuận và theo chiều nghịch:

$$
\begin{aligned}
& d x_{i}=\left(x_{i}\right)_{T}-\left(x_{i}\right)_{N} \\
& d y_{i}=\left(y_{i}\right)_{T}-\left(y_{i}\right)_{N}
\end{aligned}
$$

và tính đại lượng

$$
M_{i}=\sqrt{\left(d x_{i}\right)^{2}+\left(d y_{i}\right)^{2}}
$$

Đối với tất cả các điểm có đo góc trên đồ hình đường chuyền được nghiên cứu, góc đo thô sẽ thuộc điểm nào có đại lượng $\mathrm{M}$ nhỏ nhất. Kết thúc quá trình tìm kiếm trị đo thô trong đường chuyền và tiến hành chỉnh sửa góc đo thô được tìm thấy.

Bước 4. Kiểm tra sự có mặt và tìm kiếm cạnh đo thô

Khi không phát hiện sự tồn tại của góc đo thô ở bước 3 , tiến hành kiểm tra sự có mặt của cạnh đo thô theo quy trình sau:

* Tính các sai số khép tọa độ theo chiều thuận:

Đối với đường chuyền phù hợp, các sai số khép tọa độ được xác định theo công thức sau:

$$
\begin{aligned}
& f_{x}=x_{1}+S_{1} \cdot \cos \alpha_{1}+S_{2} \cdot \cos \alpha_{2}+\ldots+S_{n-1} \cdot \cos \alpha_{n-1}-x_{n} \\
& f_{y}=y_{1}+S_{1} \cdot \sin \alpha_{1}+S_{2} \cdot \sin \alpha_{2}+\ldots+S_{n-1} \cdot \sin \alpha_{n-1}-y_{n}
\end{aligned}
$$

Đối với đa giác đường chuyền, các sai số khép tọa độ được xác định theo công thức sau:

$$
\begin{aligned}
& f_{x}=S_{1} \cdot \cos \alpha_{1}+S_{2} \cdot \cos \alpha_{2}+\ldots+S_{n-1} \cdot \cos \alpha_{n-1} \\
& f_{y}=S_{1} \cdot \sin \alpha_{1}+S_{2} \cdot \sin \alpha_{2}+\ldots+S_{n-1} \cdot \sin \alpha_{n-1}
\end{aligned}
$$

* Tính sai số khép tọa độ tương đối theo công thức sau:

$$
\frac{1}{\Sigma S / M}
$$


Ở đây, $\Sigma S$ - là tổng chiều dài cạnh đo của đồ hình đang nghiên cứu; $M=\sqrt{f_{x}^{2}+f_{y}^{2}}$

So sánh sai số khép tọa độ tương đối tính được với hạn sai cho phép theo cấp, hạng của mạng lưới đường chuyền. Nếu vượt hạn sai, thì có thể kết luận rằng trong đồ hình đường chuyền đang nghiên cứu có tồn tại cạnh đo thô. Việc tìm kiếm cạnh đo thô được thực hiện như sau:

Tìm các cạnh mà số gia hoành độ $\Delta x_{i}=S_{i} \cdot \cos \alpha_{i}$ có dấu cùng với đại lượng $f_{x}$ và đồng thời số gia tung độ $\Delta y_{i}=S_{i} \cdot \sin \alpha_{i}$ có cùng dấu với đại lượng $f_{y}$;

Trên $m$ cạnh tìm được ở trên, chúng ta tiến hành tính các đại lượng: $\operatorname{dtg} \alpha_{j}=\left|\frac{\Delta y_{j}}{\Delta x_{j}}-\frac{f_{y}}{f_{x}}\right|$
đây $\mathrm{j}=1,2, \ldots, \mathrm{m}$.

Cạnh đo thô sẽ là cạnh có đại lượng $d \operatorname{tg} \alpha_{j}$ nhỏ nhất.

\section{Thực nghiệm}

3.1. Đối với đường chuyền phù hợp

Thực nghiệm tiến hành đối với đường chuyền phù hợp (hình 3) có các số liệu gốc và số liệu đo như bảng 1 và bảng 2 . Giả sử, góc đo $\beta_{4}$ tại điểm 4 sai số thô. Chúng ta tiến hành tìm kiếm sai thô.

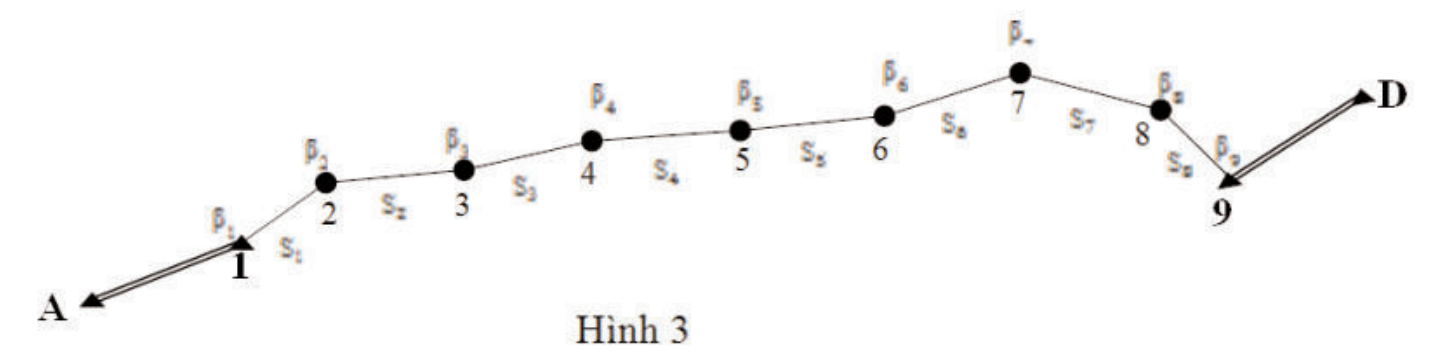

* Số liệu gốc

Bảng 1

* Số liệu đo

\begin{tabular}{|c|c|c|}
\hline Điểm & Toạ độ $\mathrm{X}(\mathrm{m})$ & Toạ độ $\mathrm{Y}(\mathrm{m})$ \\
\hline A & 2336294.650 & 569899.351 \\
\hline 1 & 2336499.363 & 570276.649 \\
\hline 2 & 23369783978 & 571137.692 \\
\hline B & 2337430.647 & 571454.425 \\
\hline
\end{tabular}

\begin{tabular}{|c|c|r|}
\hline STT & Cạnh đo $S_{1}(\mathrm{~m})$ & Góc đo $\beta_{1}\left({ }^{0}, \quad\right.$ ") \\
\hline 1 & 172.273 & $13426 \quad 13$ \\
\hline 2 & 157.342 & 2105935 \\
\hline 3 & 128.058 & 1524154 \\
\hline 4 & 171.322 & 2044618 \\
\hline 5 & 153.985 & 2144012 \\
\hline 6 & 133.283 & 1662010 \\
\hline 7 & 156.738 & 2180500 \\
\hline 8 & 143.209 & 2051407 \\
\hline 9 & & 864530 \\
\hline
\end{tabular}




\subsubsection{Tìm góc đo thô}

- Tính được các phương vị gốc như sau: $\alpha_{A 1}=61^{\circ} 31^{\prime} 01^{\prime \prime}$ và $\alpha_{9 B}=35^{\circ} 02^{\prime} 24^{\prime \prime}$

- Tính sai số khép phương vị:

$$
W_{\alpha}=1593^{\circ} 58^{\prime} 59^{\prime \prime}+(9-1) \cdot 180^{\circ}+61^{\circ} 31^{\prime} 01^{\prime \prime}-35^{\circ} 02^{\prime} 24^{\prime \prime}=27^{\prime} 36^{\prime \prime}
$$

- Hạn sai cho phép của sai số khép phương vị: $\quad\left(W_{\alpha}\right) c p= \pm 2,5.15^{\prime \prime} \cdot \sqrt{9}= \pm 1 ' 52,5^{\prime \prime}$

So sánh giữa $W_{\alpha}$ và $\left(W_{\alpha}\right) c p$ ta thấy rằng $W_{\alpha}>\left(W_{\alpha}\right) c p$. Như vậy, có góc đo thô.

Chúng tôi đã tiến hành tính tọa độ các điểm trong đường chuyền theo hai chiều thuận - nghịch, tiếp theo là tính các giá trị $\mathrm{M}_{\mathrm{i}}$, kết quả tính được thống kê ở bảng 3.

Bảng 3

\begin{tabular}{|c|c|c|c|c|c|c|c|c|c|}
\hline Điểm & \multicolumn{2}{|l|}{ Toạ độ tính theo chiều thuận } & Điểm & \multicolumn{2}{l}{ Toạ độ tính theo chiều nghịch } & Điểm & $\mathrm{dx}$ & $\mathrm{d}$ & $\mathrm{M}_{\mathrm{i}}$ \\
\hline 1 & 2336499.363 & 570276.649 & 9 & 2336978.978 & 571137.692 & 1 & 1.671 & -3.088 & 3.511123 \\
\hline 2 & 2336665.001 & 570324.000 & 8 & 2337067.700 & 571025.277 & 2 & 1.297 & -1.757 & 2.183863 \\
\hline 3 & 2336772.414 & 570438.973 & 7 & 2337103.082 & 570872.585 & 3 & 0.377 & -0.891 & 0.967476 \\
\hline 4 & 2336893.018 & 570482.025 & 6 & 2337046.676 & 570751.826 & 4 & 0.035 & 0.078 & $\underline{0.085493}$ \\
\hline 5 & 2337015.387 & 570601.929 & 5 & 2337016.311 & 570600.865 & 5 & -0.924 & 1.064 & 1.409210 \\
\hline 6 & 2337044.539 & 570753.129 & 4 & 2336892.983 & 570481.947 & 6 & -2.137 & 1.303 & 2.502914 \\
\hline 7 & 2337099.973 & 570874.337 & 3 & 2336772.037 & 570439.864 & 7 & -3.109 & 1.752 & 3.568667 \\
\hline 8 & 2337063.366 & 571026.740 & 2 & 2336663.704 & 570325.757 & 8 & -4.334 & 1.463 & 4.574268 \\
\hline 9 & 2336973.744 & 571138.410 & 1 & 2336497.692 & 570279.737 & 9 & -5.234 & 0.718 & 5.283018 \\
\hline
\end{tabular}

Theo bảng 3 , chúng ta thấy điểm 4 có giá trị $\mathrm{M}_{4}$ là nhỏ nhất. Như vậy, góc đo tại điểm 4 có chứa sai số thô. Tiến hành hiệu chỉnh góc đo thô tại điểm 4 và tiếp tục kiểm tra sự có mặt của cạnh đo thô.

\subsubsection{Tìm cạnh đo thô}

Nếu đường chuyền phù hợp ở hình 3 không có góc đo chứa sai thô, chúng ta tiến hành tìm kiếm cạnh có trị đo thô. Giả thiết, cạnh đo $S 7$ chứa sai thô có giá trị bằng $165.738 \mathrm{~m}$ (giá trị không có sai thô là: $156.738 \mathrm{~m}$ ). Chúng ta, tiến hành tìm kiếm và chứng minh cạnh đo S7 là cạnh có chứa sai thô.

Thực hiện các phép tính ở bước 4, kết quả được thống kê ở bảng 4 như sau:

Chúng ta có:

Các sai số khép tọa độ: $\mathrm{f}_{\mathrm{x}}=-2.010 \mathrm{~m} ; \mathrm{f}_{\mathrm{y}}=8.848 \mathrm{~m}$

$$
M=\sqrt{f_{x}^{2}+f_{y}^{2}}=9.037 m
$$




\section{Nghiên cúu}

- Sai số khép tọa độ tương đối:

$$
\frac{1}{\Sigma S / M}=\frac{1}{1225.210 / 9.073} \approx \frac{1}{140}
$$

Bảng 4

\begin{tabular}{|c|c|c|c|c|c|}
\hline & \multirow{2}{*}{$\begin{array}{l}\text { Góc đo } \\
\left(\begin{array}{lll}0 & \end{array}\right)\end{array}$} & \multirow{2}{*}{ 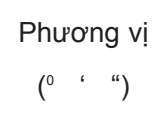 } & \multirow{2}{*}{ Chiều dài $(\mathrm{m})$} & \multicolumn{2}{|c|}{ Gia số toạ độ } \\
\hline & & & & $\Delta \times(\mathrm{m})$ & Y $(\mathrm{m})$ \\
\hline \multicolumn{6}{|l|}{ A } \\
\hline & & $\begin{array}{lll}61 & 31 & 01\end{array}$ & & & \\
\hline \multirow[t]{2}{*}{1} & $134 \quad 2313$ & & & & \\
\hline & & $\begin{array}{lll}15 & 57 & 14\end{array}$ & $\mathrm{~S} 1=172.273$ & 165.638 & 47.351 \\
\hline \multirow[t]{2}{*}{2} & 2105935 & & & & \\
\hline & & $46 \quad 56 \quad 49$ & $S 2=157.342$ & 107.413 & 114.973 \\
\hline \multirow[t]{2}{*}{3} & 1524154 & & & & \\
\hline & & 193843 & $S 3=128.058$ & 120.604 & 43.052 \\
\hline \multirow[t]{2}{*}{4} & $204 \quad 1846$ & & & & \\
\hline & & $\begin{array}{lll}43 & 57 & 29\end{array}$ & $S 4=171.322$ & 123.326 & 118.92 \\
\hline \multirow[t]{2}{*}{5} & $214 \quad 40 \quad 12$ & & & & \\
\hline & & $\begin{array}{lll}78 & 37 & 41\end{array}$ & $S 5=153.985$ & 30.362 & 150.962 \\
\hline \multirow[t]{2}{*}{6} & $\begin{array}{lll}166 & 20 & 10\end{array}$ & & & & \\
\hline & & 645751 & $S 6=133.283$ & 56.403 & 120.76 \\
\hline \multirow[t]{2}{*}{7} & $\begin{array}{lll}218 & 0500\end{array}$ & & & & \\
\hline & & $103 \quad 0251$ & $S 7=165.738$ & -37.417 & 161.459 \\
\hline \multirow[t]{2}{*}{8} & 2051407 & & & & \\
\hline & & $\begin{array}{lll}128 & 16 & 58\end{array}$ & $S 8=143.209$ & -88.724 & 112.414 \\
\hline \multirow[t]{2}{*}{9} & $86 \quad 45 \quad 30$ & & & & \\
\hline & & $\begin{array}{lll}35 & 02 & 24\end{array}$ & & & \\
\hline \multicolumn{6}{|l|}{ B } \\
\hline Tổng & & & 1225.210 & 477.605 & 869.891 \\
\hline
\end{tabular}

Nhận thấy rằng, $\frac{1}{140}$ > $\frac{1}{10000}$ (là sai số khép tương đối giới hạn của lưới khống chế đo vẽ cấp 1). Theo [1], có thể kết luận rằng đường chuyền này tồn tại cạnh đo thô. Theo bảng 4, có cạnh $\mathrm{S} 7$ và $S 8$ có các gia số tọa độ có cùng dấu với $\mathrm{f}_{\mathrm{x}}$ và $\mathrm{f}_{\mathrm{y}}$.

Tính các đại lượng: $\quad \operatorname{dtg} \alpha_{7}=\left|\frac{\Delta y_{7}}{\Delta x_{7}}-\frac{f_{y}}{f_{x}}\right|=0.087 \quad \operatorname{dtg} \alpha_{8}=\left|\frac{\Delta y_{8}}{\Delta x_{8}}-\frac{f_{y}}{f_{x}}\right|=3.135$ 
Như vậy, có thể kết luận được rằng cạnh S7 là cạnh đo thô. Điều này hoàn toàn phù hợp với giả thiết đặt ra ban đầu của bài toán.

3.2. Đối với đường chuyền đa giác khép kín

Thực nghiệm đối với đường chuyền đa giác khép kín (hình 4) có các số liệu gốc và số liệu đo như bảng 5 và bảng 6 . Giả sử, góc đo $B_{7}$ tại điểm 7 chứa sai số thô. Tiến hành tìm kiếm trị đo thô như sau:

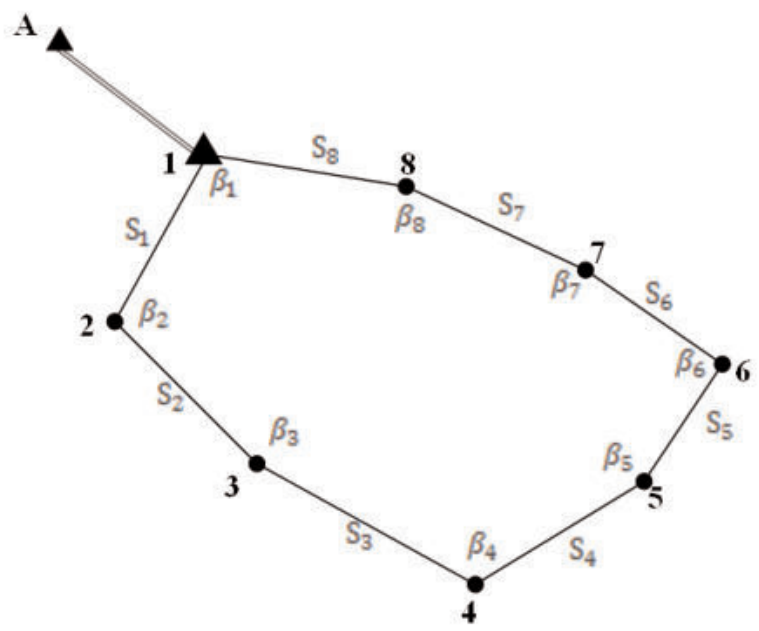

Hinh 4

* Số liệu gốc

Bảng 5

\begin{tabular}{|c|c|c|}
\hline Điểm & Toạ độ $X(\mathrm{~m})$ & Toạ độ $\mathrm{Y}(\mathrm{m})$ \\
\hline A & 2336978.978 & 571137.692 \\
\hline 1 & 2336303.284 & 571651.076 \\
\hline
\end{tabular}

* Số liệu đo

Bảng 6

\begin{tabular}{|c|c|r|}
\hline STT & Cạnh đo $S_{1}(\mathrm{~m})$ & Góc đo $\beta_{1}\left({ }^{0}, \quad\right.$ ") \\
\hline 1 & 265.742 & 873252 \\
\hline 2 & 203.373 & 983015 \\
\hline 3 & 279.528 & 1792910 \\
\hline 4 & 205.666 & 1375415 \\
\hline 5 & 198.505 & 1105057 \\
\hline 6 & 201.845 & 1054212 \\
\hline 7 & 227.087 & 1801805 \\
\hline 8 & 174.395 & $17954 \quad 46$ \\
\hline
\end{tabular}

\subsubsection{Tìm góc đo thô}

Tương tự trường hợp đường chuyền phù hợp, chúng ta tính được sai số khép phương vị và hạn sai cho phép như sau: 


$$
\begin{gathered}
W_{\alpha}=1080^{\circ} 12^{\prime} 32^{\prime \prime}-(8-2) \cdot 180^{\circ}=12^{\prime} 32^{\prime \prime} \\
\left(W_{\alpha}\right) c p= \pm 2,5.15^{\prime \prime} \cdot \sqrt{8}= \pm 1^{\prime} 46.07^{\prime \prime}
\end{gathered}
$$

So sánh giữa $W_{\alpha}$ và $\left(W_{\alpha}\right) c p$ ta thấy rằng $W_{\alpha}>\left(W_{\alpha}\right) c p$. Như vậy, có góc đo thô.

Tiến hành tính tọa độ các điểm trong đường chuyền theo hai chiều thuận - nghịch, tiếp theo là tính các giá trị $\mathrm{M}_{\mathrm{i}}$, kết quả tính được thống kê ở bảng 7 .

Bảng 7

\begin{tabular}{|c|c|c|c|c|c|c|c|c|c|}
\hline Điểm & \multicolumn{2}{|l}{ Toạ độ tính theo chiều thuận } & Điểm & \multicolumn{2}{l}{ Toạ độ tính theo chiều nghịch } & Điểm & $\mathrm{dx}$ & $\mathrm{dy}$ & $\mathrm{M}_{\mathrm{i}}$ \\
\hline 1 & 2336303.284 & 571651.076 & 1 & 2336303.284 & 571651.076 & 1 & 2.329 & 1.786 & 2.93496797 \\
\hline 2 & 2336089.238 & 571493.586 & 8 & 2336194.015 & 571786.994 & 2 & 1.740 & 0.109 & 1.74341074 \\
\hline 3 & 2335945.811 & 571637.771 & 7 & 2336051.462 & 571963.762 & 3 & 1.213 & -0.413 & 1.28138129 \\
\hline 4 & 2335750.462 & 571837.708 & 6 & 2335925.582 & 572121.546 & 4 & 0.482 & -1.124 & 1.22298814 \\
\hline 5 & 2335742.426 & 572043.217 & 5 & 2335742.694 & 572044.369 & 5 & -0.268 & -1.152 & 1.18276287 \\
\hline 6 & 2335925.031 & 572121.061 & 4 & 2335749.980 & 571838.832 & 6 & -0.551 & -0.485 & 0.73404768 \\
\hline 7 & 2336051.485 & 571963.737 & 3 & 2335944.598 & 571638.184 & 7 & 0.023 & -0.025 & $\underline{\mathbf{0}}$ \\
\hline 8 & 2336194.682 & 571787.490 & 2 & 2336087.498 & 571493.477 & 8 & 0.667 & 0.496 & 0.83120695 \\
\hline
\end{tabular}

Theo bảng 7 , chúng ta thấy điểm 7 có giá trị $\mathrm{M}_{7}$ là nhỏ nhất. Như vậy, góc đo tại điểm 7 có chứa sai số thô.

\subsubsection{Tìm cạnh đo thô}

Tương tự như đường chuyền phù hợp, chúng ta giả thiết trong đường chuyền đa giác khép kín này có cạnh đo $S_{7}$ là cạnh đo thô có giá trị là $272.087 \mathrm{~m}$ (trị đo thực là $227.087 \mathrm{~m}$ ). Chúng ta tiến hành chứng minh cạnh $S_{7}$ là cạnh đo thô.

Thực hiện các phép tính ở bước 4 , kết quả được thống kê ở bảng 8 :

Ta có:

- Các sai số khép tọa độ:

$$
\begin{aligned}
& f_{x}=28.247 m ; f_{y}=-35.074 m \\
& M=\sqrt{f_{x}^{2}+f_{y}^{2}}=45.034 m
\end{aligned}
$$

- Sai số khép tọa độ tương đối:

$$
\frac{1}{\Sigma S / M}=\frac{1}{1801.141 / 45.034} \approx \frac{1}{40}
$$


Bảng 7

\begin{tabular}{|c|c|c|c|c|c|}
\hline & \multirow{2}{*}{$\begin{array}{l}\text { Góc đo } \\
\left(\begin{array}{lll}0 & \cdots\end{array}\right)\end{array}$} & \multirow{2}{*}{ 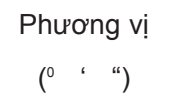 } & \multirow{2}{*}{ Chiều dài $(\mathrm{m})$} & \multicolumn{2}{|c|}{ Gia số toạ độ } \\
\hline & & & & 起 $\mathrm{X}(\mathrm{m})$ & $\Delta Y(m)$ \\
\hline \multicolumn{6}{|l|}{1} \\
\hline & & $\begin{array}{lll}216 & 20 & 41\end{array}$ & $S 1=265.742$ & -214.046 & -157.490 \\
\hline \multirow[t]{2}{*}{2} & $\begin{array}{lll}98 & 30 & 15\end{array}$ & & & & \\
\hline & & $134 \quad 50 \quad 56$ & $S 2=203.373$ & -143.427 & 144.185 \\
\hline \multirow[t]{2}{*}{3} & $\begin{array}{lll}179 & 29 & 10\end{array}$ & & & & \\
\hline & & $134 \quad 20 \quad 06$ & S3 $=279.528$ & -195.349 & 199.937 \\
\hline \multirow[t]{2}{*}{4} & $137 \quad 54 \quad 15$ & & & & \\
\hline & & $92 \quad 14 \quad 21$ & $S 4=205.666$ & -8.036 & 205.509 \\
\hline \multirow[t]{2}{*}{5} & $\begin{array}{ll}110 & 50 \quad 57\end{array}$ & & & & \\
\hline & & $\begin{array}{lll}23 & 05 & 18\end{array}$ & $\mathrm{~S} 5=198.505$ & 182.605 & 77.844 \\
\hline \multirow[t]{2}{*}{6} & $\begin{array}{lll}105 \quad 42 \quad 12\end{array}$ & & & & \\
\hline & & $\begin{array}{lll}308 & 47 & 30\end{array}$ & $S 6=201.845$ & 126.454 & -157.324 \\
\hline \multirow[t]{2}{*}{7} & $180 \quad 05 \quad 18$ & & & & \\
\hline & & $\begin{array}{lll}308 & 52 \quad 48\end{array}$ & $S 7=272.087$ & 170.787 & -211.809 \\
\hline \multirow[t]{2}{*}{8} & $17954 \quad 46$ & & & & \\
\hline & & $\begin{array}{lll}308 & 47 & 34\end{array}$ & $S 8=174.395$ & 109.259 & -135.926 \\
\hline 1 & $87 \quad 32 \quad 52$ & & & & \\
\hline Tổng & & & 1801.141 & 28.247 & -35.074 \\
\hline
\end{tabular}

Nhận thấy rằng, $\frac{1}{40} \gg \frac{1}{10000}$ (là sai số khép tương đối giới hạn của lưới khống chế đo vẽ cấp 1), theo [1] có thể kết luận rằng đường chuyền này tồn tại cạnh đo thô.Theo bảng 8 , có cạnh $S_{6}, S_{7}$ và $S_{8}$ có các gia số tọa độ tương ứng cùng dấu với fx và fy.

Tiến hành tính các đại lượng:

$$
d \operatorname{tg} \alpha_{6}=\left|\frac{\Delta y_{6}}{\Delta x_{6}}-\frac{f_{y}}{f_{x}}\right|=0.0024 ; \operatorname{dtg} \alpha_{7}=\left|\frac{\Delta y_{7}}{\Delta x_{7}}-\frac{f_{y}}{f_{x}}\right|=0.0015 ; \operatorname{dtg} \alpha_{8}=\left|\frac{\Delta y_{8}}{\Delta x_{8}}-\frac{f_{y}}{f_{x}}\right|=0.0024
$$

Như vậy, có thể kết luận được rằng cạnh $\mathrm{S} 7$ là cạnh đo thô. Điều này hoàn toàn phù hợp với giả thiết đặt ra ban đầu của bài toán.

\section{Kết luận}

- Quá trình kiểm tra sự có mặt và tìm kiếm các trị đo thô trong mạng lưới đường chuyền được tiến hành lần lượt theo tất cả các đồ hình đường chuyền cơ bản có trong mạng lưới đó.

- Các kết quả nghiên cứu ở trên cho thấy rằng mạng lưới đường chuyền có khả năng rất hạn chế trong việc tìm kiếm các trị đo thô. Do đó cần hết sức cẩn thận trong việc đưa 
các trị đo trong mạng lưới đường chuyền vào cơ sở dữ liệu trắc địa. $\bigcirc$

\section{Tài liệu tham khảo}

[1]. Bộ Tài nguyên và Môi Trường, thông tư 25_Quy định về bản đồ địa chính, ngày 19 tháng 5 năm 2014;

[2]. Hà Minh Hòa (2013). Phương pháp bình sai truy hồi với phép biến đổi xoay. Nhà xuât bản khoa học và kỹ thuật, 287 trang, Hà Nội, 2013.

[3]. Hoàng Ngọc Hà, Trương Quang Hiếu (1999), Cơ sở toán học xử lý số liệu Trắc địa, Nhà xuất bản Giao thông vận tải, Hà Nội. $\bigcirc$

\section{Summary}

\section{Methodology to detect large errors during traverse adjustment}

MSc. Luong Thanh Thach

\section{Hanoi University of Natural Resource and Environment}

Development of surveying technology; nowadays, especially GNSS launch can solve difficulties of the former methods - traverses. However, implementation of the standard GNSS network is not always feasible. Accordingly, establishing traverse by total station or theodolite is necessary because of the flexibleness. Thus, the paper presents conceptual framework to identify measurement including large error in traverse adjusting and then examines to closed line traverse and closed loop traverse. $\bigcirc$

\section{VỀ VIỆC HIỆU CHỈNH CÁC HIỆU ĐỊA THẾ.......}

(Tiếp theo trang 5)

[2]. Hà Minh Hòa (2014a). Phương pháp xử lý toán học các mạng lưới trắc địa quốc gia. Nhà Xuất bản Khoa học và Kỹ thuật, 244 trg. Hà Nội - 2014.

[3]. Hà Minh Hòa (2014b). Lý thuyết và thực tiễn của Trọng lực trắc địa. Nhà Xuất bản Khoa học và Kỹ thuật, 592 trg. Hà Nội - 2014.

[4]. Mkinen J. (2008). The treatment of permanent tide in EUREF products. Paper presented at the Symposium of the IAG Sub-commission for Europe (EUREF) in Brussels, June 17 - 21, 2008. Submitted to the proceedings.

[5]. Sacher M., Ihde J., Liebsch G., Mkinen J. (2008). EVRF2007 as Realization of the European Vertical Reference System. EUREF Symposium, June 17 - 21 2008, Brussels. O

\section{Summary}

About a correction of geopotential differences from mean tide system to zero tide system in task of the state vertical network by geopotential differences

Assoc. Prof. Dr. Sc. Ha Minh Hoa, MSc. Nguyen Thi Thanh Huong

Vietnam Institute of Geodesy and Cartography

This scientific article shows existence of systematic errors in normal height values in mean tide system under the effect of the zonal wave arisen from the attraction of the Moon, Sun and necessity of conversion of normal heights from mean tide system to zero tide system for solving the modern tasks of the high geodesy. Besides, from conservation of geopotential values in mean tide system and zero tide system there ins't necessity of conversion of a geopotential differences from mean tide system to zero tide system in case of the adjustment of the state vertical network by the geopotential differences. $\bigcirc$ 